\title{
Homogeneous selections from hyperplanes
}

\author{
Imre Bárány ${ }^{\mathrm{a}, \mathrm{b}}$, János Pach ${ }^{\mathrm{a}, \mathrm{c}}$ \\ a Rényi Institute of Mathematics, Hungarian Academy of Sciences, PO Box 127, 1364 Budapest, Hungary \\ b Department of Mathematics, University College London, Gower Street, London WC1E 6BT, England, \\ United Kingdom \\ ${ }^{\mathrm{c}}$ Chair of Combinatorial Geometry, EPFL-SB-MATHGEOM-DCG, Station 8, CH-1015 Lausanne, Switzerland
}

\section{A R T I C L E I N F O}

\section{Article history:}

Received 4 August 2011

Available online 11 November 2013

\section{Keywords:}

Hyperplanes

Hypergraphs

Intersecting simplices

\begin{abstract}
A B S T R A C T
Given $d+1$ hyperplanes $h_{1}, \ldots, h_{d+1}$ in general position in $\mathbb{R}^{d}$, let $\triangle\left(h_{1}, \ldots, h_{d+1}\right)$ denote the unique bounded simplex enclosed by them. There exists a constant $c(d)>0$ such that for any finite families $H_{1}, \ldots, H_{d+1}$ of hyperplanes in $\mathbb{R}^{d}$, there are subfamilies $H_{i}^{*} \subset H_{i}$ with $\left|H_{i}^{*}\right| \geqslant c(d)\left|H_{i}\right|$ and a point $p \in \mathbb{R}^{d}$ with the property that $p \in \triangle\left(h_{1}, \ldots, h_{d+1}\right)$ for all $h_{i} \in H_{i}^{*}$.
\end{abstract}

(C) 2013 Elsevier Inc. All rights reserved.

\section{The main result}

Throughout this paper, let $H_{1}, \ldots, H_{d+1}$ be finite families of hyperplanes in $\mathbb{R}^{d}$ in general position. That is, we assume that (1) no element of $\bigcup_{i=1}^{d+1} H_{i}$ passes through the origin, (2) any $d$ elements have precisely one point in common, and (3) no $d+1$ of them have a nonempty intersection. A transversal to these families is an ordered $(d+1)$-tuple $h=\left(h_{1}, \ldots, h_{d+1}\right) \in \prod_{i=1}^{d+1} H_{i}$, where $h_{i} \in H_{i}$ for every $i$.

Given hyperplanes $h_{1}, \ldots, h_{d+1} \subset \mathbb{R}^{d}$ in general position in $\mathbb{R}^{d}$, there is a unique simplex denoted by $\Delta=\Delta\left(h_{1}, \ldots, h_{d+1}\right)$ whose boundary is contained in $\bigcup_{1}^{d+1} h_{i}$. Clearly, this simplex is identical to the convex hull of the points

$$
v_{i}=\bigcap_{j \neq i} h_{j}, \quad i \in[d+1],
$$

where, as in the sequel, $[n]$ stands for the set $\{1,2, \ldots, n\}$.

Our main result is the following.

E-mail addresses: barany@renyi.hu (I. Bárány), pach@renyi.hu (J. Pach). 
Theorem 1.1. For every $d \geqslant 1$ there is a constant $c(d)>0$ with the following property. Given finite families $H_{1}, \ldots, H_{d+1}$ of hyperplanes in $\mathbb{R}^{d}$ in general position, there are subfamilies $H_{i}^{*} \subset H_{i}$ with $\left|H_{i}^{*}\right| \geqslant c(d)\left|H_{i}\right|$ for $i=1, \ldots, d+1$ and a point $p \in \mathbb{R}^{d}$ such that $p$ is contained in $\triangle(h)$ for every transversal $h \in \prod_{i=1}^{d+1} H_{i}$.

It follows from the general position assumption that the simplices $\Delta(h)$ in Theorem 1.1 also have an interior point in common.

It will be convenient to use the language of hypergraphs. Let $\mathcal{H}=\mathcal{H}\left(H_{1}, \ldots, H_{d+1}\right)$ be the complete $(d+1)$-partite hypergraph with vertex classes $H_{1}, \ldots, H_{d+1}$. We refer to $\mathcal{H}$ as the hyperplane hypergraph, or h-hypergraph associated with the hyperplane families $H_{1}, \ldots, H_{d+1}$. The hyperedges of $\mathcal{H}$ are the transversals of the families $H_{1}, \ldots, H_{d+1}$. Our main result can now be reformulated as follows.

Theorem 1.2. For every positive integer $d$, there is a constant $c(d)>0$ with the following property. Every complete $(d+1)$-partite h-hypergraph $\mathcal{H}\left(H_{1}, \ldots, H_{d+1}\right)$ contains a complete $(d+1)$-partite $h$-subhypergraph $\mathcal{H}^{*}\left(H_{1}^{*}, \ldots, H_{d+1}^{*}\right)$ such that $\left|H_{i}^{*}\right| \geqslant c(d)\left|H_{i}\right|$ for all $i \in[d+1]$ and $\bigcap_{h \in \mathcal{H}^{*}} \Delta(h) \neq \emptyset$.

In some sense, our theorem extends the following recent and beautiful result of Karasev [7].

Theorem 1.3. (See [7].) Assume $r$ is a prime power and $t \geqslant 2 r-1$. Let $\mathcal{H}$ be a complete $(d+1)$-partite $h$ hypergraph with partition classes of size t. Then there are vertex-disjoint hyperedges (transversals) $h^{1}, \ldots, h^{r}$ of $\mathcal{H}$ such that $\bigcap_{j=1}^{r} \Delta\left(h^{j}\right) \neq \emptyset$.

Two hyperedges (transversals) $h$ and $h^{\prime}$ of $\mathcal{H}$ are vertex-disjoint if $h_{i}$ and $h_{i}^{\prime}$ are distinct for each $i$.

Our Theorem 1.1 implies a weaker version of Karasev's theorem. Namely, the same conclusion holds with arbitrary $r$ and $t \geqslant r / c(d)$. Since $c(d)$ will turn out to be doubly exponential in $d$, our result is quantitatively much weaker than the bound $t \geqslant 4 r$ that follows from Karasev's theorem for any $r$.

Karasev's result is a kind of dual to Tverberg's famous theorem [10]. In the same sense, our result is dual to the homogeneous point selection theorem of Pach [9] (see also [8]), which guarantees the existence of an absolute constant $c_{d}>0$ with the following property. Let $X_{1}, \ldots, X_{d+1}$ be finite sets of points in general position in $\mathbb{R}^{d}$ with $\left|X_{i}\right|=n$ for every $i$. Then there exist subsets $X_{i}^{*} \subset X_{i}$ of size at least $c_{d} n$ for every $i \in[d+1]$ and a point $p \in \mathbb{R}^{d}$ such that $p \in \operatorname{conv}\left\{x_{1}, \ldots, x_{d+1}\right\}$ for all transversals $\left(x_{1}, \ldots, x_{d+1}\right) \in \prod_{i+1}^{d+1} X_{i}^{*}$. Here the assumption that the sets $X_{i}$ are of the same size can be removed (see e.g. [5]).

To establish Theorem 1.2, we need some preparation. Let $h$ and $h^{\prime}$ be two edges of the hypergraph $\mathcal{H}=\mathcal{H}\left(H_{1}, \ldots, H_{d+1}\right)$. As in (1.1), let $v_{i}$ (and $v_{i}^{\prime}$ ) denote the vertex of $\Delta(h)$ (and $\Delta\left(h^{\prime}\right)$ ) opposite to the facet contained in $h_{i}$ (and $h_{i}^{\prime}$, respectively). The edges $h$ and $h^{\prime}$ are said to be of the same type if, for each $i \in[d+1]$, the vertices $v_{i}$ and $v_{i}^{\prime}$ are not separated by either of the hyperplanes $h_{i}$ and $h_{i}^{\prime}$. We say that the h-hypergraph $\mathcal{H}$ is homogeneous if every pair of its edges is of the same type.

The heart of the proof of Theorem 1.2 is the following "same type lemma" for hyperplanes.

Lemma 1.4. For any $d \geqslant 1$, there exists a constant $b(d)>0$ with the following property. Every complete $(d+1)$-partite h-hypergraph $\mathcal{H}\left(H_{1}, \ldots, H_{d+1}\right)$ contains a complete $(d+1)$-partite subhypergraph $\mathcal{H}^{*}\left(H_{1}^{*}, \ldots, H_{d+1}^{*}\right)$ with $\left|H_{i}^{*}\right| \geqslant b(d)\left|H_{i}\right|$ for all $i \in[d+1]$ which is homogeneous.

The rest of this note is organized as follows. In Section 2, we deduce Theorem 1.2 from Lemma 1.4. In Sections 3 and 4 we present two proofs for Lemma 1.4. The first proof, which provides a better estimate for the value of the constant $b(d)$, uses duality and is based on a same type lemma for points, due to Bárány and Valtr [2] (see also [8]). The second proof is shorter, but it utilizes a far reaching generalization of the same type lemma to semi-algebraic relations of several variables, found by Fox, Gromov, Lafforgue, Naor, and Pach [5], see also Bukh and Hubard [4] for a quantitative form. The same result for binary semi-algebraic relations was first established by Alon, Pach, Pinchasi, Radoičić, and Sharir [1]. 


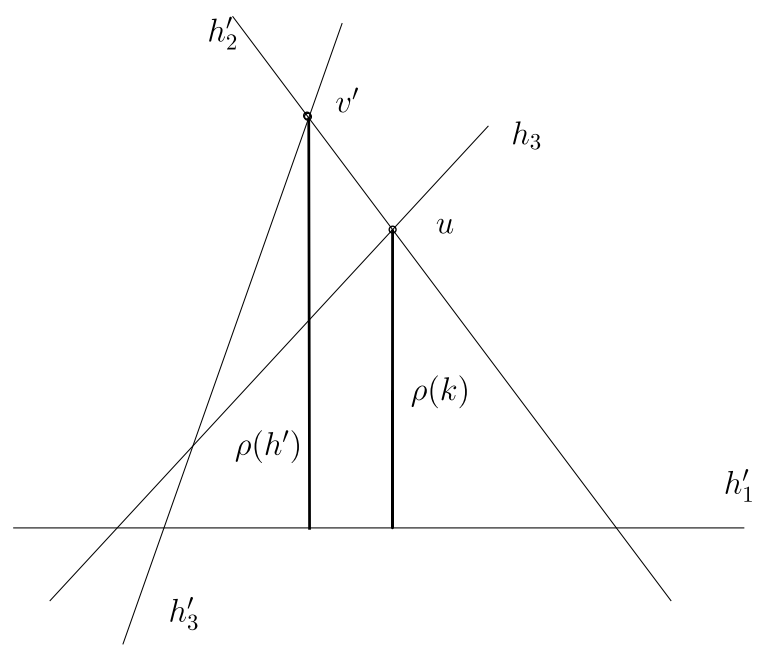

Fig. 1. Illustration for Theorem $1.2, d=2$.

\section{Proof of Theorem 1.2}

In this section, we deduce Theorem 1.2 from Lemma 1.4. The proof of the lemma is postponed to the last two sections.

Let $\mathcal{H}^{*}$ denote the complete $(d+1)$-partite subhypergraph of $\mathcal{H}$ whose existence is guaranteed by the lemma. For a fixed $h=\left(h_{1}, \ldots, h_{d+1}\right) \in \mathcal{H}^{*}$, let $h_{i}^{+}$denote the half-space bounded by $h_{i}$ that contains vertex $v_{i}$ of $\Delta(h)$, for $i \in[d+1]$. The lemma implies that, for every hyperedge $k=\left(k_{1}, \ldots, k_{d+1}\right) \in \mathcal{H}^{*}$ and for every $i$, the half-space $h_{i}^{+}$contains the vertex $u_{i}$ of $\triangle(k)$ opposite to hyperplane $k_{i}$. To prove the theorem, it suffices to establish the following claim:

$$
\bigcap_{h \in \mathcal{H}^{*}} \triangle(h) \neq \emptyset .
$$

For $h=\left(h_{1}, \ldots, h_{d+1}\right) \in \mathcal{H}^{*}$, let $\rho(h)$ denote the distance between $h_{1}$ and $v_{1}=\bigcap_{2}^{d+1} h_{j}$, and let $h^{\prime} \in \mathcal{H}^{*}$ be the edge for which $\rho(h)$ is minimal. By the general position assumption, we have $\rho\left(h^{\prime}\right)>0$. Set $v^{\prime}=\bigcap_{2}^{d+1} h_{j}^{\prime}$. We show that $v^{\prime} \in \triangle(h)$ for every $h \in \mathcal{H}^{*}$, which implies the claim. To see this, we have to verify that $v^{\prime} \in h_{i}^{+}$for every $h \in \mathcal{H}^{*}$ and for every $i$.

This is trivial for $i=1$. Suppose that $i \geqslant 2$. By symmetry, we may assume that $i=d+1$. We have to show that $v^{\prime} \in h_{d+1}^{+}$for every $h_{d+1} \in H_{d+1}^{*}$.

Assume to the contrary that $v^{\prime} \notin h_{d+1}^{+}$for some $h_{d+1} \in H_{d+1}^{*}$. Setting $k=\left(h_{1}^{\prime}, \ldots, h_{d}^{\prime}, h_{d+1}\right)$, we clearly have $k \in \mathcal{H}^{*}$. The simplices $\triangle(k)$ and $\triangle\left(h^{\prime}\right)$ share the vertex $v_{d+1}=\bigcap_{1}^{d} h_{i}^{\prime}$. As $v_{d+1} \in h_{d+1}^{+}$, by the construction, $v^{\prime} \notin h_{d+1}^{+}$implies that $h_{d+1}$ intersects the segment $\left[v_{d+1}, v^{\prime}\right]$ in a point $u$ in its relative interior, see Fig. 1. On the other hand, we know that $u=\bigcap_{2}^{d+1} k_{i}$ is the vertex of $\triangle(k)$ opposite to $h_{1}^{\prime}=k_{1}$. Thus, $u$ is closer to $h_{1}=k_{1}$ than $v^{\prime}$ is. Therefore, we obtain that $\rho(k)<\rho\left(h^{\prime}\right)$, contradicting the definition of $h^{\prime}$.

It follows from the above proof that Theorems 1.2 and 1.1 hold with $c(d)=b(d)$.

\section{A same type lemma for hyperplanes - First proof of Lemma 1.4}

Before turning to the proof of Lemma 1.4, we need some preparation. A collection of $m \geqslant d+1$ finite sets of points, $X_{1}, \ldots, X_{m} \subset \mathbb{R}^{d}$, is said to be strongly separated if every hyperplane intersects at most $d$ of the sets conv $X_{i}, i \in[m]$. This property can be rephrased in several equivalent forms; see, e.g., $[6,2,9,8]$. 
Proposition 3.1. A collection of finite point sets $X_{1}, \ldots, X_{m}$ in $\mathbb{R}^{d}$ with $m \geqslant d+1$ is strongly separated if and only if every $d+1$ of them are strongly separated.

Proposition 3.2. A collection of finite sets $X_{1}, \ldots, X_{d+1}$ in $\mathbb{R}^{d}$ is strongly separated if and only if for every subset $I \subset[d+1]$ the sets $\bigcup_{i \in I} X_{i}$ and $\bigcup_{i \in[d+1] \backslash I} X_{i}$ can be strictly separated by a hyperplane.

Two transversals $\left(x_{1}, \ldots, x_{m}\right)$ and $\left(y_{1}, \ldots, y_{m}\right) \in \prod_{i=1}^{m} X_{i}$ are said to be of the same type if the orientations of the simplices $\operatorname{conv}\left\{x_{i_{1}}, \ldots, x_{i_{d+1}}\right\}$ and $\operatorname{conv}\left\{y_{i_{1}}, \ldots, y_{i_{d+1}}\right\}$ are the same for all $1 \leqslant i_{1}<$ $i_{2}<\cdots<i_{d+1} \leqslant m$. In other words, the signs of the determinants of the matrices

$$
\left(\begin{array}{cccc}
x_{i_{1}} & x_{i_{2}} & \ldots & x_{i_{d+1}} \\
1 & 1 & \ldots & 1
\end{array}\right) \text { and }\left(\begin{array}{cccc}
y_{i_{1}} & y_{i_{2}} & \ldots & y_{i_{d+1}} \\
1 & 1 & \ldots & 1
\end{array}\right)
$$

are the same.

Proposition 3.3. A collection of finite sets $X_{1}, \ldots, X_{m}$ in $\mathbb{R}^{d}$ with $m \geqslant d+1$ is strongly separated if and only if every pair of transversals of $X_{i}(i \in[m])$ are of the same type.

As usual, we say that a set of points $X \subset \mathbb{R}^{d}$ is in general position if no $d+1$ elements of $X$ lie on a hyperplane. We need the same type lemma of Bárány and Valtr [2] for points.

Theorem 3.4. (See [2].) For every positive integer $d$ and every $m \geqslant d+1$, there is a constant $c(d, m)>0$ with the following property. Let $X_{1}, \ldots, X_{m}$ be a collection of pairwise disjoint finite point sets in $\mathbb{R}^{d}$ such that their union is in general position. Then there exist subsets $X_{i}^{*} \subset X_{i}$ with $\left|X_{i}^{*}\right| \geqslant c(d, m)\left|X_{i}\right|$ for all $i \in[m]$ such that the collection $X_{1}^{*}, \ldots, X_{m}^{*}$ is strongly separated.

Now we turn to the proof of Lemma 1.4. We use the standard duality between points $a \in \mathbb{R}^{d} \backslash\{0\}$ and hyperplanes $h \subset \mathbb{R}^{d}$ with $0 \notin h$. Every hyperplane not passing through the origin 0 is of the form

$$
h=\left\{x \in \mathbb{R}^{d}: a \cdot x=1\right\},
$$

with a unique $a \in \mathbb{R}^{d} \backslash\{0\}$. Conversely, every $a \in \mathbb{R}^{d} \backslash\{0\}$ gives rise to a unique hyperplane $h$ via (3.1). By the general position assumption, no element of $\bigcup_{i=1}^{d+1} H_{i}$ passes through the origin. For any $i \in$ $[d+1]$, let $A_{i}$ denote the set of points dual to the hyperplanes in $H_{i}$ via the standard duality (3.1).

Applying Theorem 3.4 to the sets $A_{0}=\{0\}, A_{1}, \ldots, A_{d+1}$, we obtain a collection of subsets $A_{0}^{*}=\{0\}, A_{1}^{*} \subset A_{1}, \ldots, A_{d+1}^{*} \subset A_{d+1}$ with $\left|A_{i}^{*}\right| \geqslant c(d, d+2)\left|A_{i}\right|$ for all $i \in[d+1]$ such that all $(d+2)$-transversals of them are of the same type. The sets of hyperplanes dual to the elements of $A_{1}^{*}, \ldots, A_{d+1}^{*}$, denoted by $H_{1}^{*}, \ldots, H_{d+1}^{*}$, form a complete $(d+1)$-partite h-hypergraph $\mathcal{H}^{*}\left(H_{1}^{*}, \ldots, H_{d+1}^{*}\right)$, which is a subhypergraph of the original hypergraph $\mathcal{H}$.

Claim 3.5. The h-hypergraph $\mathcal{H}^{*}$ is homogeneous.

Proof. We show that, given $h_{i} \in H_{i}^{*}$ and $h_{j}, k_{j} \in H_{j}^{*}$ (where $j \neq i$ ), $h_{i}$ does not separate the points $v=\bigcap_{j \neq i} h_{j}$ and $u=\bigcap_{j \neq i} k_{j}$. By symmetry, it suffices to prove this in the case $i=d+1$.

Consider the simplices $\Delta_{0}=\Delta\left(h_{1}, \ldots, h_{d+1}\right), \Delta_{1}=\Delta\left(k_{1}, h_{2}, \ldots, h_{d+1}\right), \Delta_{2}=\Delta\left(k_{1}, k_{2}, h_{3}, \ldots\right.$, $\left.h_{d+1}\right), \ldots, \triangle_{d}=\triangle\left(k_{1}, \ldots, k_{d}, h_{d+1}\right)$. Let $u_{i}$ be the vertex opposite to $h_{d+1}$ in $\triangle_{i}$. We have $u_{0}=v$ and $u_{d}=u$. Obviously, it is sufficient to verify that $h_{d+1}$ does not separate $u_{i-1}$ and $u_{i}$ for $i \in[d]$, see Fig. 2.

Again, by symmetry, it is enough to consider the case $i=1$. Assume that $h_{1}$ and $k_{1}$ are given by the equations $a_{1} \cdot x=1$ and $a_{1}^{\prime} \cdot x=1$, respectively. Set $a(t)=(1-t) a_{1}+t a_{1}^{\prime}$ for $t \in[0,1]$ and let $h(t)$ be the hyperplane with equation $a(t) \cdot x=1$, and let $\Delta(t)$ be the corresponding simplex (if it exists, which is not entirely clear at the moment) with vertex $u(t)$ opposite to $h_{d+1}$. 


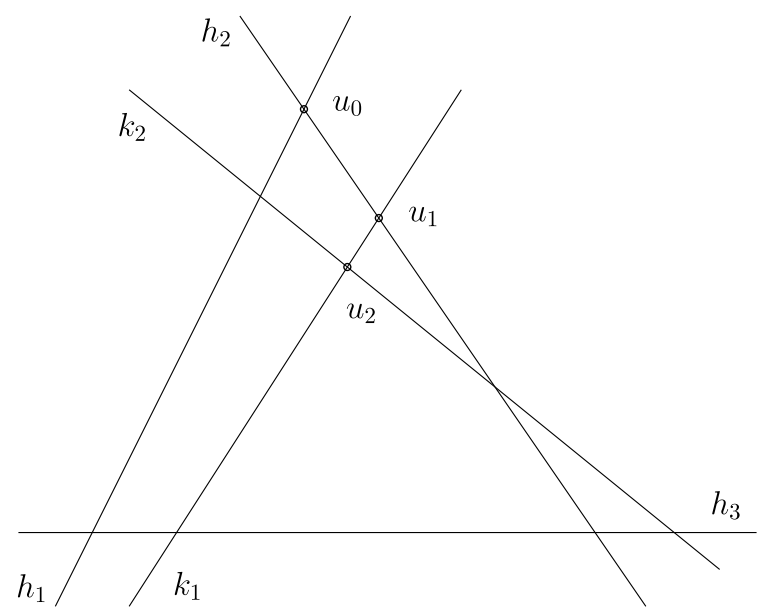

Fig. 2. Illustration for Claim 3.5, case $d=2$.

We move $h_{1}$ to $k_{1}$ by the homotopy $h(t)$ and check how $u(t)$ behaves. The common vertex of $\triangle_{0}$ and $\Delta_{1}$ is $z=\bigcap_{2}^{d+1} h_{i}$. The segment $\left[z, u_{0}\right]$ is an edge of $\Delta_{0}$. We define the half-line $L=\left\{z+\lambda\left(u_{0}-\right.\right.$ $z): \lambda>0\}$.

We will show that $h(t) \cap L$ is a single point for every $t \in[0,1]$. This will complete the proof, because $h(0) \cap L=u_{0}, h(1) \cap L=u_{1}$, and $L$ lies completely on one side of $h_{d+1}$. Suppose the contrary and let $T \in[0,1]$ be the smallest $t \in[0,1]$ such that for all $\tau \in[0, t), h(\tau) \cap L$ is a single point but $h(t) \cap L$ is not. (General position implies that $T>0$.) This can happen in two different ways: either $h(T)$ contains $z$ or $h(T)$ becomes parallel to $L$.

Case 1. $z \in h(T)$. Then the equations

$$
a(T) \cdot x=1, \quad a_{2} \cdot x=1, \quad \ldots, \quad a_{d+1} \cdot x=1
$$

have a common solution, namely $z$. The points $a(T) \in \operatorname{conv} A_{1}^{*}, a_{2} \in A_{2}^{*}, \ldots, a_{d+1} \in \operatorname{conv} A_{d+1}^{*}$ lie on the same hyperplane, namely on $\{x: x \cdot z=1\}$. But this is impossible, as $A_{1}^{*}, \ldots, A_{d+1}^{*}$ satisfy Theorem 3.4.

Case 2. $h(T)$ is parallel to $L$ or, equivalently, to $u_{0}-z$. Then $u_{0}-z$ is a solution of the equations

$$
a(T) \cdot x=0, \quad a_{2} \cdot x=0, \quad \ldots, \quad a_{d} \cdot x=0,
$$

and also to $a_{0} \cdot x=0$ where $a_{0}=0$. Therefore, the points $a_{0} \in A_{0}^{*}, a(T) \in \operatorname{conv} A_{1}^{*}, \ldots, a_{d} \in \operatorname{conv} A_{d}^{*}$ lie on the same hyperplane, namely on the one with equation $x \cdot\left(u_{0}-z\right)=0$. This is again impossible.

In view of the above arguments, in Lemma 1.4 and in Theorems 1.1 and 1.2 , one can take $c(d)=$ $b(d)=c(d, d+2)=2^{-(d+1) 2^{d}}$, where $c(d, d+2)$ comes from Theorem 3.4.

\section{Semi-algebraic relations - Second proof of Lemma 1.4}

A real semi-algebraic set in $\mathbb{R}^{d}$ is the locus of all points that satisfy a given finite Boolean combination of polynomial equations and inequalities in the $d$ coordinates. We say that the description complexity of such a set is at most $s$ if in some representation the number of equations and inequalities is at most $s$ and each of them is of degree at most $s$. Such a representation is usually called quantifier-free. Note that semi-algebraic sets can also be defined using quantifiers involving additional variables, but these quantifiers can always be eliminated (see [3]).

Let $H_{1}, \ldots, H_{m}$ be families of semi-algebraic sets of constant description complexity, and let $R$ be an $m$-ary relation on $\prod_{1}^{m} H_{i}$. We assume that $R$ is also semi-algebraic, in the following sense. We 
associate each $h \in H_{i}$ with a point $\bar{h} \in \mathbb{R}^{d_{i}}$ (say, with the point whose coordinates are the coefficients of the monomials in the polynomial inequalities defining $h$ ). We say that $R$ is a semi-algebraic m-ary relation if its corresponding representation

$$
\bar{R}=\left\{\left(\bar{h}_{1}, \ldots, \bar{h}_{m}\right) \in \mathbb{R}^{d_{1}+\cdots+d_{m}} \mid h_{1} \in H_{1}, \ldots, h_{m} \in H_{m}\left(h_{1}, \ldots, h_{m}\right) \in R\right\}
$$

is a semi-algebraic set.

We need the following result of Fox et al. [5]. Its proof is based on the case $m=2$, established by Alon et al. [1].

Theorem 4.1. Let $\alpha>0$, let $H_{1}, \ldots, H_{m}$ be finite families of semi-algebraic sets of constant description complexity, and let $R$ be a fixed semi-algebraic m-ary relation on $H_{1} \times \cdots \times H_{m}$ such that the number of m-tuples that are related (resp. unrelated) with respect to $R$ is at least $\alpha \prod_{i=1}^{m}\left|H_{i}\right|$. Then there exists a constant $c^{\prime}>0$, which depends on $\alpha, m$ and on the maximum description complexity of the sets in $H_{i}(i \in[m])$ and $R$, and there exist subfamilies $H_{i}^{*} \subseteq H_{i}$ with $\left|H_{i}^{*}\right| \geqslant c^{\prime}\left|H_{i}\right|(i \in[m])$ such that $\prod_{1}^{m} H_{i}^{*} \subset R\left(\operatorname{resp} . \prod_{1}^{m} H_{i}^{*} \cap R=\emptyset\right)$.

Proof of Lemma 1.4. We apply Theorem 4.1 with $m=d+1$ for the families of hyperplanes $H_{i}, i \in$ $[d+1]$. As in the previous section, we associate each hyperplane $h_{i} \in H_{i}$ with its dual vector $a_{i} \in$ $\mathbb{R}^{d} \backslash\{0\}$ satisfying

$$
h_{i}=\left\{x \in \mathbb{R}^{d}: a_{i} \cdot x=1\right\} .
$$

As in (1.1), given a $(d+1)$-tuple of hyperplanes $\left(h_{1}, \ldots, h_{d+1}\right) \in \prod_{1}^{d+1} H_{i}$, for every $i \in[d+1]$, let $v_{i}=\bigcap_{j \in[d+1] \backslash\{i\}} h_{j}$. That is, $v_{i}$ is the unique solution of the equations $a_{j} \cdot x=1$ for $j \in[d+1] \backslash\{i\}$. Using the assumption that the hyperplanes are in general position, we have $v_{i} \notin h_{i}$. Therefore, $v_{i}$ must lie in one of the open half-spaces bounded by $h_{i}$, depending on $\operatorname{sign}\left(a_{i} \cdot v_{i}-1\right)$.

Define $2^{d+1}$ different $(d+1)$-ary relations on $\prod_{1}^{d+1} H_{i}$, depending on the sign pattern

$$
\left(\operatorname{sign}\left(a_{1} \cdot v_{1}-1\right), \ldots, \operatorname{sign}\left(a_{d+1} \cdot v_{d+1}-1\right)\right) .
$$

For example, one of these relations is the relation $R^{+}$, according to which $\left(h_{1}, \ldots, h_{d+1}\right)$ are related if and only if $\operatorname{sign}\left(a_{i} \cdot v_{i}-1\right)>0$ for all $i \in[d+1]$. Obviously, each $(d+1)$-tuple $\left(h_{1}, \ldots, h_{d+1}\right) \in \prod_{1}^{d+1} H_{i}$ is related by precisely one of the above relations. Therefore, for at least one relation $R$, the number of $(d+1)$-tuples related with respect to $R$ is at least $\frac{1}{2^{d+1}} \prod_{i=1}^{d+1}\left|H_{i}\right|$. Hence, if $R$ is a semi-algebraic relation, then Lemma 1.4 follows directly from Theorem 4.1 .

To see that the above relations are semi-algebraic, it is sufficient to observe the following. Let $A$ be the $d$ by $d$ matrix whose columns are $a_{2}, \ldots, a_{d+1}$, and write $A_{k}$ for the matrix obtained from $A$ by replacing its $k$ th column by a column whose each entry is 1 . Since $v_{1}$ is the unique solution of the equations $a_{j} \cdot x=1$ for $j \in[d+1] \backslash\{1\}$, by Cramer's rule we obtain that the $k$ th coordinate of $v_{1} \in \mathbb{R}^{d}$ is $\operatorname{det} A_{k} / \operatorname{det} A$. Thus, we have

$$
a_{1} \cdot v_{1}-1=\sum_{k=1}^{d} a_{1 k} \frac{\operatorname{det} A_{k}}{\operatorname{det} A}-1,
$$

where $a_{1 k}$ denotes the $k$ th component of $a_{1}$. Consequently,

$$
\operatorname{sign}\left(a_{1} \cdot v_{1}-1\right)=\operatorname{sign}\left[\operatorname{det} A\left(\sum_{k=1}^{d} a_{1 k} \operatorname{det} A_{k}\right)-(\operatorname{det} A)^{2}\right] .
$$

The last expression in square brackets is a polynomial in the variables $a_{i k}, i \in[d+1], k \in[d]$. Analogously, $\operatorname{sign}\left(a_{2} \cdot v_{2}-1\right), \ldots, \operatorname{sign}\left(a_{d+1} \cdot v_{d+1}-1\right)$ can be written as the sign of a polynomial, which implies that the above relations are indeed semi-algebraic.

This proof gives a weaker constant in Lemma 1.4 and consequently in Theorem 1.1. Namely, using a quantitative version of a weaker form of Theorem 4.1 obtained by Bukh and Hubard [4], we obtain 
$c(d)=b(d)=3^{-(d+1) 3^{d^{2}+d+1}}$. Note that Fox et al. [5] used Theorem 4.1 to establish a much stronger structure theorem for semi-algebraic relations.

\section{Acknowledgments}

Part of this work was done at Centre Interfacultaire Bernoulli, EPFL, Lausanne. Research partially supported by NSF Grant CCF-08-30272, by Swiss National Science Foundation Grants 200020-144531 and 200021-137574, by ERC Advanced Research Grant 267165, by Hungarian Science Foundation EuroGIGA Grants OTKA NN 102029 and OTKA 78439. The authors are grateful to R. Radoičić for his valuable suggestions.

\section{References}

[1] N. Alon, J. Pach, R. Pinchasi, M. Radoičić, M. Sharir, Crossing patterns of semi-algebraic sets, J. Combin. Theory Ser. A 111 (2005) 310-326.

[2] I. Bárány, P. Valtr, Positive fraction Erdős-Szekeres theorem, Discrete Comput. Geom. 19 (1998) 335-342.

[3] S. Basu, R. Pollack, M.-F. Roy, Algorithms in Real Algebraic Geometry, 2nd edition, Springer, Berlin, 2006.

[4] B. Bukh, A. Hubard, Space crossing numbers, in: Symposium on Computational Geometry, ACM Press, 2011, pp. 163-170.

[5] M. Gromov, V. Lafforgue, A. Naor, J. Pach, Overlap properties of geometric expanders, J. Reine Angew. Math. 671 (2012) 49-83.

[6] J.E. Goodman, R. Pollack, R. Wenger, Geometric transversal theory, in: J. Pach (Ed.), New Trends in Discrete and Computational Geometry, Springer-Verlag, New York, 1991.

[7] R.N. Karasev, Dual central point theorems and their generalizations, arXiv:0909.4915, and also Mat. Sb. 199 (2008) 1459-1479.

[8] J. Matoušek, Lectures on Discrete Geometry, Springer, Heidelberg, 2002.

[9] J. Pach, A Tverberg-type result on multicolored simplices, Comput. Geom. 1 (1998) 71-76.

[10] H. Tverberg, A generalization of Radon's theorem, J. Lond. Math. Soc. 41 (1966) 123-128. 